\title{
LA CONSPIRATION DES ÉMOTIONS
}

\section{Patrick Boucheron}

Éditions de Minuit | « Critique »

2014/11 n $810 \mid$ pages 869 à 880

ISSN 0011-1600

ISBN 9782707328199

Article disponible en ligne à l'adresse :

https://www.cairn.info/revue-critique-2014-11-page-869.htm

Distribution électronique Cairn.info pour Éditions de Minuit.

(C) Éditions de Minuit. Tous droits réservés pour tous pays.

La reproduction ou représentation de cet article, notamment par photocopie, n'est autorisée que dans les limites des conditions générales d'utilisation du site ou, le cas échéant, des conditions générales de la licence souscrite par votre établissement. Toute autre reproduction ou représentation, en tout ou partie, sous quelque forme et de quelque manière que ce soit, est interdite sauf accord préalable et écrit de l'éditeur, en dehors des cas prévus par la législation en vigueur en France. Il est précisé que son stockage dans une base de données est également interdit. 


\title{
La conspiration des émotions
}

\author{
Katsumata Shizuo \\ Ikki \\ Coalitions, ligues et révoltes dans \\ le Japon d'autrefois [1982] \\ Introduction, traduction et notes \\ de Pierre-François Souyri \\ (avec la collaboration \\ de Inde Tadao et Nakajima Keiichi)
}

\author{
Paris, CNRS Éditions, \\ coll. «Réseau Asie », \\ 2011,270 p.
}

"Les hommes en foule ne sauraient se passer de maître », affirmait Gustave Le Bon en $1895^{1}$. Mais que se passe-t-il lorsqu'on s'écarte de la foule ? Échappe-t-on si aisément à ce «besoin de chef »dont Yves Cohen a écrit récemment l'histoire implacable ? Son Siècle des chefs, vaste fresque transnationale décrivant la production sociale du leadership, butait sur l'autoritarisme de 1968 - alors commencerait selon lui une autre histoire, " un siècle de la foule sans maître, qui n'est rien d'autre que la foule contre le maître ${ }^{2} »$. Reste à comprendre pourquoi la philosophie politique qui s'autorise de cette histoire récente demeure avant tout soucieuse de rendre raison du consentement et de l'obéissance. Au risque parfois d'entretenir une fascination pour le commandement, dont Giorgio Agamben a entrepris l'archéologie. Car si cette dernière vise l'archè, on doit se souvenir que le terme désigne en grec à la fois l'ordre et le principe, le commandement et le commencement. De cette homologie structurale découle le prestige de l'origine ${ }^{3}$. Les historiens savent bien par ailleurs

1. G. Le Bon, Psychologie des foules [1895], Paris, PUF, 1963, p. 130 .

2. Y. Cohen, Le Siècle des chefs. Une histoire transnationale du commandement et de l'autorité (1890-1940), Paris, Éd. Amsterdam, 2013, p. 815.

3. G. Agamben, Qu'est-ce que le commandement?, Paris, Payot \& 
que le privilège de l'antériorité est le plus solide des principes de légitimité : ainsi dans les mouvements de résistance, où devient chef celui qui sait faire reconnaître le fait qu'il a parlé, ou agi, avant les autres ${ }^{4}$.

C'est entendu : au commencement est le commandement. Mais quel est le moyen d'y contrevenir ? Sans doute en empruntant des pentes contraires à la philosophie politique contemporaine, qui sous-estime systématiquement la capacité des acteurs à agir dans le dos du pouvoir, à feinter avec lui, à construire patiemment leur droit à l'indifférence. C'est le cas, notamment, de la philosophie d'Agamben, pour lequel l'horizon théologico-politique éclaire d'une aveuglante clarté le paradigme moderne ${ }^{5}$. Pour impressionnante que soit cette archéologie de la scission de souveraineté entre règne et gouvernement - et elle l'est particulièrement pour le médiéviste qui partage avec le philosophe la lecture de certaines de ses sources patristiques - on ne peut ignorer la critique de Georges Didi-Huberman : "Ce qui disparaît, dans cette féroce lumière du pouvoir, n'est autre que la moindre image ou lueur de contre-pouvoir ${ }^{6}$. » Frêle, fugace, intermittente - elle n'en dessine pas moins les trajectoires de constellations $^{7}$ auxquelles on ne peut se montrer attentif que si l'on adopte le point de vue de ce que Roberto Esposito appelle l'immunité - cette immunité qui est toujours une réaction à la communauté $^{8}$. Envisager le point de vue des ingouvernables nécessite donc de faire écart par rapport à une tradition de

Rivages, coll. « Bibliothèque Rivages », 2013, p. 15.

4. J.-Cl. Monod, Qu'est-ce qu'un chef en démocratie ? Politiques du charisme, Paris, Éd. du Seuil, 2012, p. 76 (citant notamment les travaux de Laurent Douzou sur la Résistance française à l'appui d'une réflexion sur la phénoménologie de l'autorité chez Alexandre Kojève).

5. G. Agamben, Le Règne et la Gloire. Pour une généalogie théologique de l'économie et du gouvernement, Homo sacer II, 2 [2007], Paris, Éd. du Seuil, 2008.

6. G. Didi-Huberman, Survivance des lucioles, Paris, Éd. de Minuit, 2009, p. 77.

7. Je fais ici allusion à l'essai du collectif «Mauvaise troupe » Constellations : trajectoires révolutionnaires du jeune $21^{e}$ siècle, Paris, Éd. de L'éclat, 2014.

8. R. Esposito, Communauté, immunité, biopolitique. Repenser les termes du politique [2008], Paris, Les Prairies ordinaires, 2010. 
philosophie politique des plus intimidantes. Faut-il pour cela se transporter nécessairement jusqu'à Zomia, ou la leçon de James Scott porte-t-elle partout où les observateurs acceptent d'accompagner son pas de côté ${ }^{9}$ ?

Pour tenter de répondre à cette question, partons d'une expérience singulière de désorientation, relatée dans un texte étrange et profond d'Ivan Illich intitulé « La culture de la conspiration ». Illich y raconte notamment comment il avait, à Yokohama, entrepris de parler de paix. Pour expliquer à son auditoire japonais la différence entre la philia grecque, qui lie les hommes, et la pax romaine, qui se fige en terre, il fait détour vers un idéogramme évidemment intraduisible, $f u$-dô, dont on lui dit qu'il peut être traduit, ou plus exactement décrit ainsi : "l'incomparable fraîcheur qui naît du mélange d'un sol particulier avec les eaux appropriées ${ }^{10}$. » Mais cette évidence olfactive de l'appartenance - proche en somme de ce que Jean-Christophe Bailly a appelé dans Le Dépaysement "l'émotion de la provenance ${ }^{11}$ »- qui est d'ici et pas d'ailleurs, s'oppose à ce geste qui va se confondre, en Occident, depuis l'époque paléochrétienne, avec l'idée de pax : la conspiratio, c'est-à-dire non seulement le fait de respirer le même air, de pouvoir se sentir, mais aussi de mélanger ses souffles.

Voici donc ce que le conférencier occidental croit avoir à dire à ses hôtes japonais : la paix ne naît pas d'un mélange de la terre et des eaux en un lieu particulier, d'un milieu dirait ici Augustin Berque ${ }^{12}$, mais de cette «intimité exigeante » du partage des souffles qui, elle, peut s'éprouver partout où se rassemblent les hommes ${ }^{13}$. Et Ivan Illich de rappeler qu'il y a

9. J. Scott, Zomia. Ou l'art de ne pas être gouverné [2009], Paris, Éd. du Seuil, 2013. Voir ici même l'entretien avec James Scott réalisé par Nils Gilman et Nicolas Guilhot, et traduit par Marielle Macé.

10. I. Illich, "La culture de la conspiration » [1998], La Perte des sens, Paris, Fayard, 2004, p. 348. Voir sur ce point le grand livre de Watsuji Tetsurô, Fudô. Le milieu humain, Paris, CNRS Éditions, 2011, traduit et commenté par Augustin Berque.

11. J.-C. Bailly, Le Dépaysement. Voyages en France, Paris, Éd. du Seuil, 2011, p. 8.

12. A. Berque, Milieu et identité humaine. Notes pour un dépassement de la modernité, Paris, Donner lieu, 2010.

13. I. Illich, « La culture de la conspiration », op. cit., p. 349. 
trois mots pour dire le baiser en latin : paternel ou conjugal, il est le tendre basium ; amoureux, il devient le lascif suavium ; il est osculum lorsqu'il se fait pacifique et politique. Dans la liturgie paléochrétienne, l'osculum est le moment fort de la célébration de l'eucharistie, c'est-à-dire non seulement l'union dans l'Esprit saint mais la production d'une atmosphère commune, respirable par tous, faisant du mystère une co-respiration, soit, au sens propre, une conspiration. Évidemment, les Pères de l'Église y mirent rapidement bon ordre : Tertullien s'inquiétait pour les matrones, et on euphémisa bientôt l'osculum en osculum pacis puis en simple pax. C'est pourquoi, poursuivait Ivan Illich, les communes médiévales, formes politiques de l'émancipation laïque $\mathrm{au} \mathrm{xII}^{\mathrm{e}}$ siècle, naissaient comme conspiratio et se fixaient contre conjuratio. La conspiration ne suffit pas - il convenait de la figer en un serment solennel, public, pris en commun, et cette tension constitutive fait de la ville une conjuratio conspirativa. C'est donc qu'elle souffle le chaud et le froid, qu'elle s'invente dans la chaleur des souffles et se maintient dans la solennelle roideur du serment écrit, car jurer en commun oblige toujours à lever les yeux au ciel - voyez le Serment du Jeu de paume de David. Dieu serait-il toujours le garant des pactes ? "Ici, je flaire un rat. Mon nez me dit que "quelque chose est pourri" dans l'État de l'Occident. Au cours du $\mathrm{II}^{\mathrm{e}}$ millénaire, l'utilisation de Dieu comme témoin pour sacraliser le contrat social a créé le cadre dans lequel il est possible d'abuser de la pax comme d'un idéal justifiant l'imposition de notre type d'ordre au monde entier ${ }^{14}$. »

Ivan Illich avait le nez fin. Son odorat subtil reconnaissait ici ce qu'il y a, aujourd'hui encore, de plus insistant dans la philosophie politique du contemporain. On remarquera au passage que la période médiévale lui sert alors fréquemment de ressource d'intelligibilité : que l'on songe à l'archéologie du serment proposée par Giorgio Agamben sous le titre Le Sacrement du langage ${ }^{15}$, très redevable aux analyses fondamentales d'Irène Rosier-Catach sur la parole efficace ${ }^{16}$, et

14. Ibid., p. 352.

15. G. Agamben, Le Sacrement du langage. Archéologie du serment [2008], Paris, Vrin, 2009.

16. I. Rosier-Catach, La Parole efficace. Signe, rituel, sacré, Paris, Éd. du Seuil, 2004. 
qui rappelle la nature fondamentalement contractuelle des constructions institutionnelles médiévales, à un moment où la pensée scolastique du pacte n'est pas encore nouée à la nature démocratique du gouvernement ${ }^{17}$. Que l'on songe également à la réévaluation en cours de la modernité politique de la forme communale, qui aurait tout intérêt à prendre appui sur la théorisation wébérienne de la conjuratio ${ }^{18}$, dès lors que celle-ci permet de penser l'efficace de la sacralisation du politique ${ }^{19}$. Autant de questions que le texte étrange et pénétrant d'Ivan Illich, penseur iconoclaste et fin médiéviste, aborde avec une subtilité déconcertante. On choisira plutôt de le suivre ici dans sa capacité de détour, accompagnant son pas de côté japonais pour dérouter le confort de nos certitudes.

La traduction récente d'un livre passionnant nous en fournit une occasion des plus heureuses : Ikki. Coalitions, ligues et révoltes dans le Japon d'autrefois de Katsumata Shizuo. On la doit au talent et à l'énergie de Pierre-François Souyri, pour qui l'histoire de ce qu'on appellera, faute de mieux, le « Moyen Âge japonais » sert à inquiéter la rassurante généalogie de la modernité occidentale, c'est-à-dire sa capacité à universaliser son point de vue particulier sur le monde ${ }^{20}$. Dans une postface inédite donnée à la réédition de son livre majeur, Le Monde à l'envers, décrivant la période troublée que connaît l'archipel nippon de la fin du $\mathrm{XII}^{\mathrm{e}}$ siècle (période de Kamakura) à la fin du Xvi ${ }^{\mathrm{e}}$ siècle (époque Sengoku), Souyri revient sur l'étrange commensurabilité des «féodalités » japonaise et occidentale ${ }^{21}$. Des châteaux, des

17. F. Foronda (éd.), Avant le contrat social. Le contrat politique dans l'Occident médiéval, $X I I{ }^{e}-X V^{e}$ siècle, Paris, Publications de la Sorbonne, 2011 ; P. de J. Olivi, Traité des contrats, présentation, édition critique et traduction de S. Piron, Paris, Les Belles Lettres, 2012.

18. F. Hulak, «L'avènement de la modernité : la commune médiévale chez Max Weber et Émile Durkheim », Archives de philosophie, vol. 76, $\mathrm{n}^{\circ} 4,2013$, p. 553-569.

19. P. Boucheron, "Religion civique, religion civile, religion séculière. L'ombre d'un doute ", Revue de synthèse, vol. 134, n 2, 2013, p. 161-183.

20. Voir notamment P.-F. Souyri, Nouvelle histoire du Japon, Paris, Perrin, 2010 (et notamment p. 280-283 sur « la naissance des Ikki »).

21. Id., «De la comparaison entre les sociétés médiévales d'Occident 
troubles, des chevaliers et des samouraïs : difficile de résister à la tentation de cette ressemblance déplacée. Elle induit pourtant de fausses évidences qu'il s'agit de déjouer patiemment en tentant de « construire des comparables », selon le mot d'ordre bien connu de Marcel Détienne ${ }^{22}$.

L'histoire du Japon a plus et mieux à offrir que le miroir rassurant du grand récit de la modernité étatique que l'Occident a constitué en savoir exclusif. Elle peut au contraire être vue " comme un "dépays", pour reprendre l'expression du cinéaste Chris Marker, en ce sens qu'il nous dépayse et nous déstabilise, jamais là où on l'attend, qu'il nous oblige à reformuler nos questionnements, qu'il déplace nos catégories de pensée $^{23} »$. Précieuse est de ce point de vue la contextualisation historiographique que Pierre-François Souyri propose du travail de Katsumata Shizuo dans son introduction (p. 13-39). Katsumata fait partie des historiens qui, dans le cours des années 1970-1980, ont pris appui sur la riche tradition des études folkloriques pour fonder une école japonaise d'anthropologie historique proposant une histoire sociale remarquablement attentive aux pratiques.

Dans le cas présent - soit l'étude des soulèvements paysans et, d'une manière générale, de la contestation populaire dans le Japon médiéval - il s'agissait d'abord d'affronter une tradition historiographique qui, sous le double impact des émeutes du riz de 1918 et de l'influence du marxisme sur les historiens japonais, avait construit un récit volontiers dramatique où les révoltes villageoises $d u x^{e}$ et du début du $\mathrm{XVI}^{\mathrm{e}}$ siècle réussissaient à imposer un rapport de force favorable aux communautés paysannes. Et ce jusqu'à ce que de nouveaux seigneurs de la guerre parviennent à restaurer un État fort à la fin du $\mathrm{Xvi}^{\mathrm{e}}$ siècle, le régime de Tokugawa précipitant une reféodalisation de la société japonaise, elle-même

et du Japon », Histoire du Japon médiéval. Le monde à l'envers [rééd. de Le Monde à l'envers. La dynamique de la société médiévale, Paris, Maisonneuve \& Larose, 1998], Paris, Perrin, coll. « Tempus », 2013, p. 417-432.

22. M. Détienne, Comparer l'incomparable, Paris, Éd. du Seuil, 2000, p. 41 et suivantes.

23. P.-F. Souyri, «La modernité japonaise dans tous ses états », Le Débat, n 153, 2009, p. 89. 
ébranlée par une nouvelle vague d'ikki qui ressurgit aux xviII et $\mathrm{XIX}^{\mathrm{e}}$ siècles pour mettre à bas le shôgunat. S'il avait eu le grand mérite de contrecarrer la tradition d'inspiration confucianiste de ce que l'on appelle au Japon « l'histoire correcte », laquelle déniait aux soulèvements populaires toute forme de rationalité en n'y voyant qu'émotions informes, ce grand récit ne rendait pas compte des permanences structurales de l'ikki, depuis le fonctionnement réglé des sociétés monastiques $\mathrm{du} \mathrm{XII}^{\mathrm{e}}$ siècle jusqu'aux soubresauts les plus violents des émeutes paysannes du XIx ${ }^{\mathrm{e}}$ siècle.

C'est à reconstituer la grammaire anthropologique de cette pratique de pouvoir en deçà même de son fonctionnement potentiellement subversif que s'attache le livre de Katsumata Shizuo. Voici peut-être ce qui est le plus passionnant, mais aussi le plus déroutant, pour son lecteur occidental : il voit défiler, sur une très longue durée, moines, guerriers et paysans, moins comme des personnages historiques que comme des acteurs de théâtre qui viendraient tour à tour endosser un rôle dont le répertoire est fixé depuis longtemps ${ }^{24}$. Car il s'agit bien, comme on va le voir, d'une histoire de masques et de travestissements, mais aussi de répertoire d'actions sur la scène politique. Ce n'est pas le moindre charme de ce livre constamment surprenant, ne serait-ce que dans sa démarche même qui, comme il est apparemment d'usage chez les historiens japonais, procède par petites touches et "par déplacements, avec un ordre qui serait caché avant d'être suggéré ${ }^{25}$ », sans qu'une affirmation théorique abrupte ne vienne jamais la figer.

Comment définir l'ikki ? Avant d'être une forme de protestation collective, les ikki désignaient un mode de gouvernement fondé sur l'organisation horizontale de coalitions

24. Voir sur ce point les remarques de Guillaume Carré dans la note critique qu'il a donnée de cet ouvrage dans la revue Cipango. Cahiers d'études japonaises, «Le Japon et le fait colonial, II », n 19, 2012, [http://cipango.revues.org/1723].

25. Je reprends ici une expression tirée de la présentation d'ensemble, par Hiroyuki Ninomiya et Pierre-François Souyri, d'un ensemble d'articles sur "L'histoire du Japon sous le regard japonais » (Annales HSS, 50-2, 1995, p. 232). On y trouvait déjà un article de Katsumata Shizuo, «Ikki. Conjurations et révoltes dans la société médiévale japonaise », p. 373-394. 
d'égaux. Unanimité des esprits, égalité des droits, solidarité entre les membres : telles sont les valeurs qui fondent l'ikki, et que le japonais classique rend par les mots ichimi dôshin, « même goût, même cœur ». C'est que le moment où le groupe s'institue est particulier : il s'agit toujours de répondre à l'urgence d'une nécessité. "Organisations exceptionnelles formées en vue d'un objectif déterminé et mues par lui », les $i k k i$ sont «finalement assez proches de ce que l'on appelle un "mouvement" dans le langage contemporain » (p. 47). Parce qu'ils ne peuvent se dissoudre qu'une fois l'objectif atteint - c'est-à-dire, le plus souvent, une fois leur point de vue reconnu par une autorité supérieure - les ikki forment des ententes provisoires mais impérieuses. Que font les députés du tiers état lors du serment du Jeu de paume, jurant de ne jamais se séparer sinon «par la force des baïonnettes »? Ils font ikki. Car ainsi que le remarque justement PierreFrançois Souyri dans sa présentation (p. 14, n. 1), il n'est pas plus absurde d'user des catégories japonaises pour rendre compte de l'histoire européenne que de pratiquer l'opération inverse, qui nous semble toujours plus spontanément légitime ${ }^{26}$.

Comme dans le fameux tableau de David en effet, où les conjurés lèvent les yeux au ciel pour prendre Dieu à témoin de leur serment collectif, c'est dans le recours au sacré que se constitue la force de l'ikki. «Boire ensemble l'eau sacrée » (ichimi jinzui) est une des expressions les plus usuelles dans les sources de la pratique, jusqu'au $x^{2} I^{\mathrm{e}}$ siècle, pour dire la constitution d'ikki. Elle désigne le rituel par lequel les conjurés se rassemblaient dans l'enceinte du sanctuaire shintô local afin d'écrire un serment, de le jurer, d'en contresigner le document, puis de le brûler et d'en verser les cendres dans l'eau sacrée qui devenait ainsi « habitée par les dieux »

26. On peut penser, dans le même ordre d'idées, à la manière dont Gabriel Martinez-Gros prend appui sur la théorie de l'histoire universelle d'Ibn Khaldûn pour éclairer des événements de l'histoire européenne postérieure, contrevenant à cette croyance fondamentale : «pour pasticher un célèbre hadith du Prophète, l'Occident explique et n'est pas expliqué » (G. Martinez-Gros, Ibn Khaldûn et les sept vies de l'Islam, Arles, Actes Sud, 2006, p. 232). Du même auteur, voir aussi Brève histoire des empires. Comment ils surgissent, comment ils s'effondrent, Paris, Éd. du Seuil, 2014. 
(yorube no mizu) et qu'ils buvaient ensuite tour à tour (p. 75). Il est inutile sans doute d'insister sur l'extraordinaire richesse de sens de ce rituel qui conjugue tous les usages, du plus pragmatique au plus magique, des pratiques de l'écrit avec des conduites d'oralité et de commensalité produisant ce que Marcel Mauss (cité fréquemment par Katsumata) appelait un « fait social total ». Il s'articule d'ailleurs avec d'autres manifestations collectives dont Katsumata Shizuo propose l'anthropologie : ainsi de l'habitude de «faire sonner le métal », c'est-à-dire d'accompagner le serment d'un tintement d'objets métalliques : " les guerriers frappaient leur sabre, les moines une cloche, les femmes un miroir» (p. 82), ou de la pratique du défilé protestataire.

De là la nature festive de l'ikki, expliquant aussi le fait " qu'il existe un rapport étroit entre le fait de constituer un ikki et de se masquer » (p. 93). Katsumata consacre de longues pages au travestissement et à la métamorphose des apparences, qui marquent toujours « la forte détermination des protestataires » (p. 171). Les kimonos oranges, le grand chapeau en paille de riz (kasa), le manteau de pluie (mino) ont aussi une dimension religieuse éclairée par les études de l'ethnographe Orikuchi Shinobu : ces vêtements transformaient les révoltés en tengu, les dieux rédempteurs du folklore japonais, ce qui légitimait par avance les destructions et les violences nécessaires à la "correction du monde » (p. 187). Pourtant, et c'est là l'aspect le plus saillant du livre, les formes protestataires de l'ikki ne sont rien d'autre que le retournement, ou la subversion, d'une pratique ordinaire de gouvernement.

En effet, ce sont d'abord les conseillers d'État qui, dès le XIII $^{\mathrm{e}}$ siècle, font ikki autour du régent shôgunal pour mieux l'aider à gouverner. Quant aux assemblées monastiques, elles adoptaient des procédures similaires de prise de décision. S'inspirant des préceptes bouddhiques, l'organisation communautaire des monastères du Japon médiéval repose donc sur deux principes qui ne sont contradictoires qu'en apparence : les décisions sont prises à la majorité des voix, mais elles sont ensuite proclamées à l'unanimité. "En d'autres termes, il est interdit aux tenants de la position minoritaire de continuer à camper sur leurs positions contre l'avis majoritaire » (p. 65). L'étude comparée des pratiques 
délibératives est sans doute l'un des grands chantiers à venir d'une histoire décloisonnée des pouvoirs médiévaux ${ }^{27}$; on voit ici combien elle gagnerait à s'ouvrir à la comparaison structurale avec des sociétés lointaines ${ }^{28}$, ne serait-ce que pour compliquer la généalogie occidentale de la maior pars qui s'imposa lentement aux pratiques du suffrage ${ }^{29}$.

"Il est clair que ces dispositions ont été prises à l'unanimité des esprits et qu'il n'y a pas de chef. Si les autorités supérieures exigeaient que nous désignions un chef, nous ne donnerions pas suite » (p. 120) : on trouve ce type de formules dans les serments d'assemblées monastiques et c'est pourquoi les participants disposent leurs signatures sur les pactes comme les baleines d'une ombrelle déployée : les révoltés paysans de l'époque d'Edo reprennent cette pratique, et notamment au $\mathrm{XIX}^{\mathrm{e}}$ siècle où les contestations paysannes se teintent de millénarisme pour œuvrer au « redressement du monde » (p. 157). Il y a donc dans la pratique même de l'ikki un principe fondamentalement anti-autoritaire, un art de ne pas se donner de chef. Et peu importe alors que l'exigence politique des ikki demeure strictement formalisée par une économie morale que Katsumata présente comme intangible : il s'agit pour l'essentiel d'obtenir un édit de «politique vertueuse » (tokusei), c'est-à-dire des actes de grâces, portant avant tout sur l'abolition des dettes. Reste que ce qu'il y a de plus éminemment subversif dans l'ikki est la forme même de l'action collective.

De ce point de vue, l'arme suprême des révoltés demeure le déguerpissement - non pas la fuite individuelle du sauvequi-peut, mais une conduite collective de l'ikki dans le cadre d'une coalition d'habitants d'un domaine féodal. Lorsqu'on lit dans les sources japonaises que les paysans coalisés ont entrepris de "tirer, étaler les bambous » (sasa wo hiku) (p. 192), il faut sans doute entendre qu'ils ont couvert de

27. Voir sur ce point la récente et belle synthèse de M. Hébert, Parlementer. Assemblées représentatives et échanges politiques en Europe occidentale à la fin du Moyen Âge, Paris, Éd. de Boccard, 2014.

28. Déjà esquissée dans M. Détienne (éd.), Qui veut prendre la parole ?, Paris, Éd. du Seuil, 2003 («Le genre humain », p. 40-41).

29. O. Christin, Vox populi. Une histoire du vote avant le suffrage universel, Paris, Éd. du Seuil, 2014, p. 41 et suivantes. 
broussailles ou de feuilles de bambou les entrées des maisons mais aussi les champs. Ici, l'obstacle tactique se conjugue avec la dimension religieuse (car les branches de bambou sont utilisées comme réceptacles pour accueillir les divinités). Échapper à l'emprise du pouvoir en bloquant l'accès aux ressources et en partant se réfugier dans la montagne : telle est la principale hantise des seigneurs qui, au même moment en Europe, se constituent en entrepreneurs de regroupement de population (congregatio populorum). Sans doute retrouve-t-on également la situation décrite par James Scott dans Zomia, rappelant que la défection - dès lors qu'elle est collective et organisée - constitue la plus redoutable des ripostes à la domination : ainsi lit-on par exemple dans L'Histoire d'une cour malaise datant $\mathrm{du} \mathrm{xvII}^{\mathrm{e}}$ siècle : " C'est la coutume des rois que de se nommer eux-mêmes rois s'ils ont des ministres et des sujets : s'il n'y a pas de sujets, qui rendra hommage au roi ${ }^{30}$ ? »

Faire $i k k i$, c'est se rendre ingouvernables. C'est aussi retourner contre le pouvoir la force de la conjuration des émotions. En lisant le livre de Katsumata Shizuo, on rêve d'une autre histoire des révoltes médiévales, terrain sensiblement délaissé par l'historiographie actuelle ${ }^{31}$. Celle-ci demeure fascinée par la puissance de cohésion sociale d'un dominium dont elle exagère la robustesse et la compacité. La théorie théologico-politique de l'ecclesia a remplacé aujourd'hui le recours au mode de production féodal comme explication totalisante d'une société passée qui sert avant tout d'appui à un rêve d'ordre social, d'obéissance politique et de croyance religieuse. Elle néglige de ce fait l'inventivité politique des laboratoires urbains et monastiques où s'éprouve ce que Jacques Dalarun appelle des «essais de démocratie médiévale ${ }^{32} »$. Elle ne prend pas suffisamment au sérieux la feinte, l'ironie, la résistance, en prêtant aux sociétés passées

30. Cité par J. Scott, "La montagne et la liberté », Critique internationale, vol. $2, \mathrm{n}^{\circ} 11,2001$, p. 91.

31. Voir cependant les travaux récents de X. Nadrigny, Information et opinion publique à Toulouse à la fin du Moyen Âge, Paris, École des Chartes, 2013, p. 199 et suivantes.

32. J. Dalarun, Gouverner c'est servir. Essai de démocratie médiévale, Paris, Alma, 2012. 
(ou lointaines) une capacité d'adhésion crédule dont on se pense affranchi. Comme le rappelle avec force Bruno Latour, la croyance dans la croyance des autres est la manière dont les Modernes s'assurent du fétiche de leur propre savoir : «Nous croyons que nous savons. Nous savons que les autres croient $^{33}$.»

Le « rat» que flairait Ivan Illich provient aussi de là. Plus encore peut-être que la mobilisation, la dispersion est un enjeu politique pour aujourd'hui. La capacité à répondre, ou à ne pas répondre, à venir ou pas, à s'y retrouver ou non - là se joue l'autonomie. Faire ikki : comment se rassembler, comment se séparer ? "Il ne faut pas durer, il ne faut pas avoir part à quelque durée que ce soit. Cela fut entendu en ce jour exceptionnel : personne n'eut à donner un ordre de dispersion. On se sépara par la même nécessité qui avait rassemblé l'innombrable. On se sépara instantanément, sans qu'il y eût de reste, sans que soient formées ces séquelles nostalgiques par lesquelles s'altère la manifestation véritable en prétendant persévérer en groupes de combat. Le peuple n'est pas ainsi. Il est là, il n'est plus là ; il ignore les structures qui pourraient le stabiliser ${ }^{34}$. »

Patrick BOUCHERON

33. B. Latour, Enquête sur les modes d'existence. Une anthropologie des Modernes, Paris, La Découverte, 2012, p. 178.

34. M. Blanchot, La Communauté inavouable, Paris, Éd. de Minuit, 1983, p. 56. 